\title{
Practice based course of Information Technology Service Management for BSc students
}

\author{
Krisztina ERDÉLYI
}

Abstract. Currently IT systems are primarily observed as sets of services, for example finance services can be accessed via the Internet. The IT background of an enterprise has strategic importance in its activity, but it is also important, that IT helps the enterprise supporting course of business really from the background. To implement this kind of conception, IT Infrastructure Library (ITIL) provides practical advices.

The paper describes the topic of an optional course which introduces BSc students to the subject of IT Service Management the way that it can be used as a practice supplement beside theory courses of IT Service Management.

Key words and phrases: IT service management, monitoring, configuration management, education.

ZDM Subject Classification: Q65.

\section{IT as a service}

Currently IT systems are primarily observed as sets of services. (Bank services can be accessed via Internet, and it seems a good idea to provide web hosting where people can provide their own websites.)

IT Service Management is a discipline for managing and operating IT systems which centres customers. Its most important principle is to determine what IT can contribute to the success of a business. This idea deliberately is the opposite of the former so called technology-centred approach which emphasised the interoperation of IT management and business. The next quotation provides a good illustration of the philosophy of IT Service Management.

Copyright (C) 2010 by University of Debrecen 


$$
\text { "tmcs-erdelyi" — 2010/11/14 — 21:59 — page 230 — \#2 }
$$

"Providers of IT services can no longer afford to focus on technology and their internal organization, they now have to consider the quality of the services they provide and focus on the relationship with customers." [1]

The greatest challenge of IT executive managers is to cooperate with the business managers to provide high quality IT services.

\subsection{IT Service Management frameworks}

Many frameworks have been developed to implement IT service management. Control Objectives for Information and related Technology (COBIT), Information Technology Infrastructure Library (ITIL) and Microsoft Operations Framework (MOF) are the three mostly used frameworks. They usually collect together best practises and then systematise them. They help IT managers to understand the relationship between business and IT and provide guidance linked to organising, implementing and measuring IT processes.

The differences between these frameworks stem from, firstly, that the approach of tackling problems and tasks are different. Since variant organisations worked out their own unique model, consequently they have their own characteristics and specialities. Secondly, differences exist because of a targeted audience. It is important whether an enterprise is a small or medium or operates on a worldwide scale.

The Control Objectives for Information and related Technology (COBIT) was created by the Information Systems Audit and Control Association (ISACA), and the IT Governance Institute (ITGI) in 1996. COBIT provides managers, auditors, and IT users with a set of generally accepted measures, indicators, processes and best practices to assist them in maximising any benefits derived through the use of information technology whilst developing appropriate IT governance and control in organisational contexts [2]. The Information Technology Infrastructure Library (ITIL) gives detailed descriptions of a number of important IT practices and provides comprehensive checklists, tasks and procedures that any IT organisation can modify according to its needs. ITIL is published in a series of books, each of which covers an IT management topic [3]. Microsoft Operations Framework (MOF) 4.0 delivers practical guidance for everyday IT practices and activities, helping users establish and implement reliable, cost-effective IT services. It is based on ITIL but aimed at small businesses that do not want to use (and buy) the whole ITIL suite [4]. 


$$
\text { "tmcs-erdelyi" — 2010/11/14 — 21:59 — page } 231 \text { — \#3 }
$$

Practice based course of Information Technology Service Management for BSc students 231

This paper focuses on ITIL methodology. However the whole IT services subject will be kept in mind. Other theorems and technologies will be mentioned in the 3 th section (Developing the Course).

\subsection{Lifecycles of ITIL}

ITIL is guidance for control, development and maintenance of IT infrastructures. It contains a set of descriptions of best practices which have become a standard. Almost every multinational computer technology corporation has made its own ITIL based software family. In them there are some independently usable elements such as monitoring, provisioning, backups, security management and account management software. ITIL v3 is the most currently version. ITIL v2 was revised, and in version 3 the service lifecycle approach is in the mainstream. Comparing to the version 2, version 3 differs only its approach but it covers everything which is in version 2 .

Most importantly, "a detailed comparison between ITIL v3 and v2 reveals that all the main processes belonging to ITIL v2 are still present. In many instances, however, ITIL v3 offers revised and enhanced process descriptions" [5]. Within ITIL there are five stages of service lifecycle. The lifecycle starts with determining and analysing the business requirements in Service Strategy and Service Design. It migrates into a live environment within Service Transition, and reaches Service Operation and Continual Service Improvement.

Improving services means subsequent design which indicates the further transition of the software. After transition the renewed software is being operated until the next, improved version is complete. Hence, a continual cycle exists in the life time of services. Figure 1 shows the role of the five stages in ITIL service lifecycle.

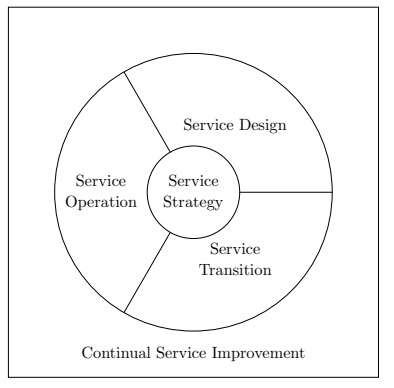

Figure 1. ITIL Life Cycle Model
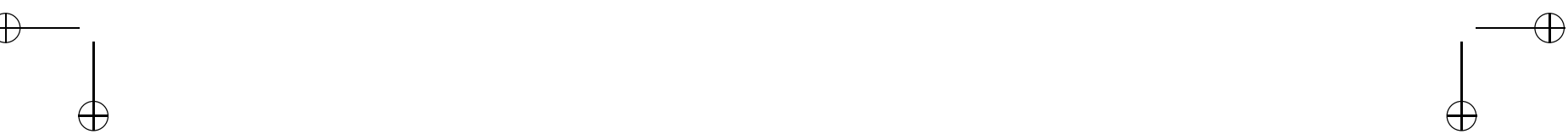


$$
\text { "tmcs-erdelyi" — 2010/11/14 — 21:59 — page 232 — \#4 }
$$

\subsection{Processes, activities and functions}

ITIL offers guidance as to how organisations can improve their processes to work more effectively. Activity is a set of actions designed to achieve a particular result. For example network monitoring is an activity "to detect events and to ensure that the current status is known" [6].

Activities form processes. According to ITIL official definition: a process is "a structured set of activities designed to accomplish a specific objective" [7]. A process takes one or more defined inputs and turns them into defined outputs. A process may include any of the roles, responsibilities, tools and management controls required to reliably deliver the outputs.

The goal of 'Service Strategy' is to provide guidance on how to design, develop and implement Service Management. So the key processes are Financial Management (managing the service provider's budgeting, accounting and charging requirements) and Service Portfolio Management (deciding on a strategy to serve customers and to develop a service provider's offerings and capabilities). Service Design is responsible for the functions that support the service lifecycle, risk management, secure IT infrastructures, environments, applications and data/information resources and measurement methods and metrics.

'Service Design' runs the following processes to implement these objectives:

- Service Catalogue Management (providing vital information for all other Service Management processes);

- Service Level Management (negotiating Service Level Agreements with the customers and to design services in accordance with the agreed service level targets);

- Risk Management (identifying, assessing and controlling risks);

- Capacity Management (ensuring that the capacity of IT services and the IT infrastructure is able to deliver the agreed service level targets in a cost effective and timely manner);

- Availability Management (defining, analyzing, planning, measuring and improving all aspects of the availability of IT services).

'Service Transition' delivers services that are required by a business into operational use whilst ensuring that any new or changed services can be used in accordance with the requirements and constraints specified within the service requirements. Key processes in this lifecycle are:

- Change Management (controlling the lifecycle of all changes);
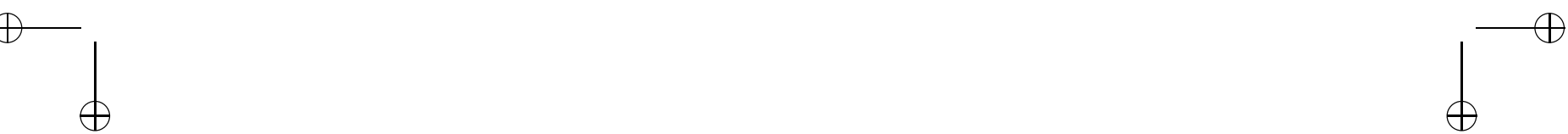


$$
\text { "tmcs-erdelyi" — 2010/11/14 — 21:59 — page 233 — \#5 }
$$

- Service Asset and Configuration Management (maintaining information about Configuration Items required to deliver an IT service, including their relationships);

- Knowledge Management (gathering, analysing, storing and sharing knowledge and information within an organisation).

The key activities of 'Service Operation' are monitoring and control of IT systems and license management. It belongs to service desk as the most important function. Event (detectable or discernible occurrence) management, incident (unplanned interruption to an IT service) management and problem (problem is an unknown cause of one or more incidents) management take place via service desk.

The most important and the oldest function of ITIL is the service desk, namely customers inform the service provider, when something works in an unusual way or under a predefined level. An unplanned interruption to an IT Service, or a reduction in the quality of an IT Service, are each called incidents. The service must be returned as fast as possible. To handle the cases appropriately means not only ending the incident, but if necessary, the whole service should be revised, known as continual service improvement.

Figure 2 shows the links to main processes of Service Operation

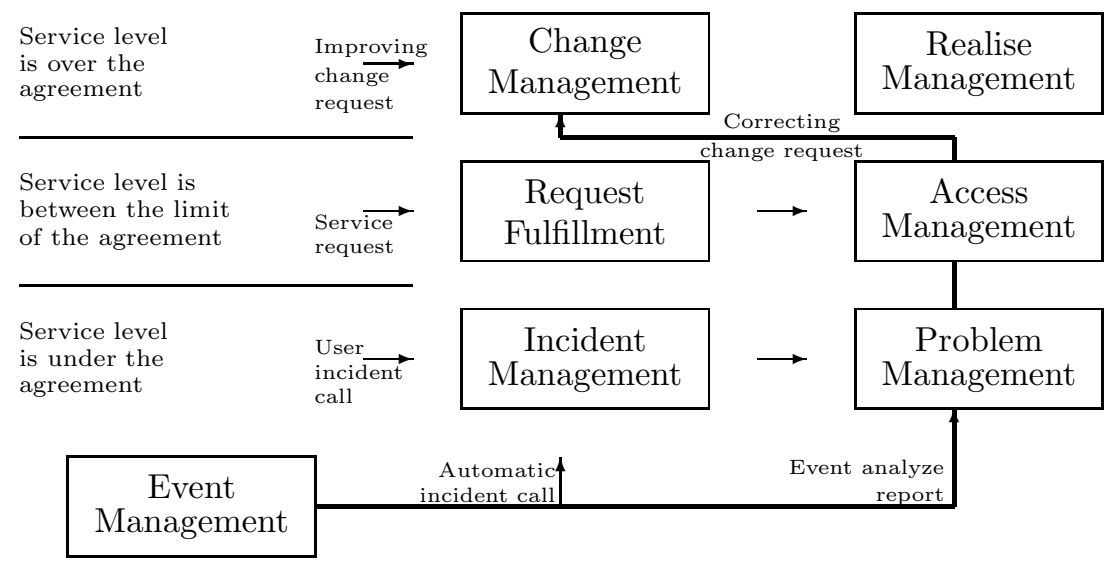

Figure 2. Main processes of Service Operation
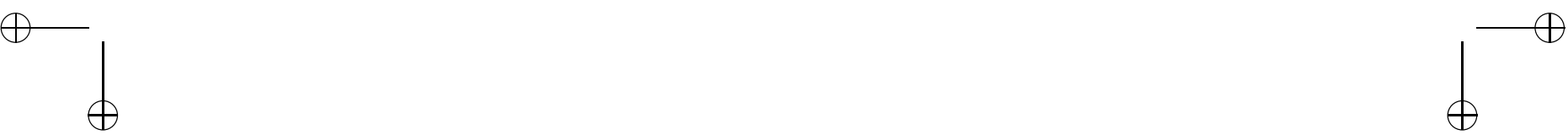


$$
\text { "tmcs-erdelyi" — 2010/11/14 — 21:59 — page 234 — \#6 }
$$

\subsection{Monitoring}

Proactive thinking is highlighted beside reactivity in ITIL, hence service providers try to act before an incident occurs and they want to avoid the potential incidents. The tool for proactive thinking is monitoring. Observing hardware, software and various networks to reveal cases that may not cause incidents, but may become an incident in the future completes the monitoring process. For example, operators may recognise an almost full hard drive, software slowing down or a bottleneck in a segment of the network. Monitoring is categorised as a process in Service Operation by ITIL with the following definition: "Repeated observation of a configuration item, IT service or process to detect events and to ensure that the current status is known" [6]. Monitoring the current status and documenting it is vital for meeting and checking Service Level Agreements (SLA). The agreement is signed by a service provider and its customer and defines the aim and the quality of the services. To verify the compliance to a SLA it contains values expressed in numbers. For example, it defines the accepted length and frequent of outage of a server.

ITIL defines a 'problem' as the cause of one or more incidents. Monitoring is used to discover any problem and to observe the system. For example, network bottlenecks and overloads can be discovered via network monitoring. From the service provider's point of view, it is not enough if a service works sufficiently. It needs to be worked within budget so a service provider seeks a more cost effective way of operation. Capacity management deals with cost effective solutions it in Service Design. Executive managers may decide to develop certain parts of the infrastructure according to the monitoring results. Figure 3 shows monitoring and its relationship to the ITIL processes.

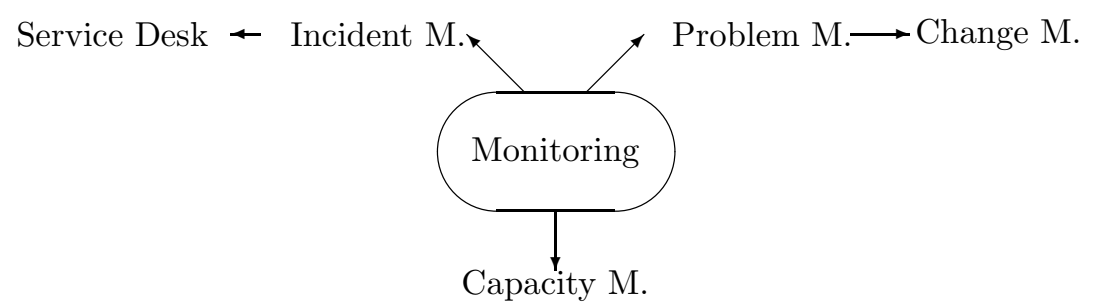

Figure 3. Monitoring and its relationship to the ITIL Processes 


$$
\text { "tmcs-erdelyi" — 2010/11/14 — 21:59 — page 235 — \#7 }
$$

Practice based course of Information Technology Service Management for BSc students

\subsection{Configuration management}

Implementation of IT services and enterprise decision support requires precise information. Managers need to know about hardware and software items owned by their enterprise, and licensing information. Managers analyse the stored data before making a decision, for example, before developing a network system they check where a bottleneck is in the current system and which server is the most overloaded one. It is advised to store all information about an IT service in one repository. For this reason a so called Configuration Management Database is built.

ITIL uses the term Configuration Item (CI) for all components that are controlled in order to provide IT services. These items can be IT services, hardware, software, people, buildings or formal documents. Information about each $\mathrm{CI}$ is recorded in a Configuration Record. Configuration Management Database (CMDB) is a kind of repository to store configuration records. Each CMDB stores attributes to configuration items and relationships between configuration items.

The goals of creating a configuration management database are the following [8]:

- Account for all the IT assets and configurations within the organisation and its services;

- Provide accurate information on configurations and their documentation to support all the other Service Management processes;

- Provide a sound basis for Incident Management, Problem Management, Change Management and Release Management.

- Provide verification of the configuration records against the infrastructure and correct any exceptions.

Configuration Management is a process which is responsible for the maintenance of information necessary to provide IT services. Its three main activities are control, integration and decision support. If configuration records reflect reality managers are able to keep control on the IT infrastructure. The integration of all process related to configuration decreases the number of errors. Moreover, making the right decision is simpler because of the unified, comprehensive data handling. 


$$
\text { "tmcs-erdelyi" — 2010/11/14 — 21:59 — page 236 — \#8 }
$$

Figure 4 shows an example of configuration management and its relationship to the ITIL processes.

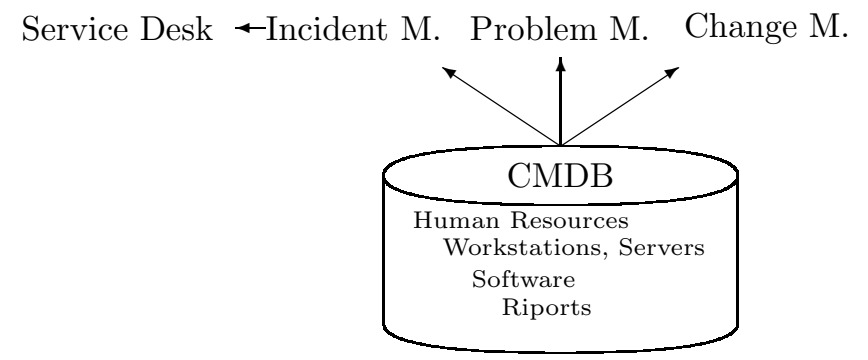

Figure 4. Configuration management and its relationship to the ITIL processes

Configuration management facilitates the work of service desk and incident management by automatically filling incident records and helping retrieving related data. Configuration management also provides the result of risk analysis for problem management. Information related to a change can be retrieved from the configuration management database before initiating change to a service component. Configuration management also handles the notification to be sent to customers about any changes being made. The new state will be perceived at once in a good configuration management system.

To summarise, a goal of the basic concept of ITIL is that an IT organisation can provide quality, continual improving services. Certainly it is important to implement it cost effectively. Achieving wide range of knowledge needs cooperation among many people in the context of modern systems.

\section{Significance of education of IT Service Management}

As previously discussed, IT service management is a part of everyday functioning in IT supported organisations. If effective, people do not recognise its existence. However, IT students ought to be familiar with the events occurring in the background of IT services. They will meet experts in IT Services, and as customers, additionally for example when they go to a bank or subscribe to an Internet service. The significance of IT Service Science education is underpinned by reviewing extant research next. 


$$
\text { "tmcs-erdelyi" — 2010/11/14 — 21:59 — page } 237 \text { — \#9 }
$$

\subsection{Summary of extent researches}

ITIL was developed for the United Kingdom government in the 1980s [3]. It moved from there and was firstly used in Europe. The standard ISO/IED 20000 normalised IT Service Management and is based on ITIL. Scientific articles related to educational topics were published mainly in Australia and New-Zealand. In [9] ITIL was shown to be effective:

"We found that both customer satisfaction and operational performance improve as the activities in the ITIL framework increases. Increased use of the ITIL framework is therefore likely to result in improvements to customer satisfaction and operational performance."

The introduction of some part of ITIL is described in [10]. It underpins by quotations that organisational management had a great motivation to implement ITIL:

"To summarize the motivation of the cases investigated in this study, all managers support the view that ITIL enables standardization of IT service management processes and terminology throughout the organisation and that such standardization is vital to ensure a consistent and reliable level of service to the business."

According to [11] "a new and growing area of academic study called Services Science is evolving", but it adds that "despite the significant growth of ITSM practice in industry, little scholarly work exists on this topic".

\subsection{Industrial training or academic education}

Teaching the topic of IT service management mostly occurs in industrial contexts. Most training centres offer preparation courses in ITIL certification tests. Multinational computer technology corporations offer courses related to their own software. The topic of ITIL in academic education appears in relatively small numbers compared to industrial settings. In Hungarian Higher Education courses related to IT Service Management was introduced very recently. A good example is $[12]$.

Contemporary research into teaching of IT service management exists in Victoria University, Australia [13]. They use the industrial certification system and the related training courses. They gives a table to compare the benefits and pitfalls of industrial trainings (Table 1). 
Table 1. Benefits and pitfalls of industrial trainings

\begin{tabular}{|c|c|}
\hline Strengths & Weaknesses \\
\hline Adding Value to degree programs & Exist to support training industry \\
\hline Work related experience & Proprietary nature \\
\hline $\begin{array}{l}\text { Practical rather than just } \\
\text { theoretical focus }\end{array}$ & $\begin{array}{l}\text { Lack of educational } \\
\text { rigor }\end{array}$ \\
\hline $\begin{array}{l}\text { "Up-to-Date" nature of } \\
\text { certification programs }\end{array}$ & $\begin{array}{c}\text { Often lacks "realworld" } \\
\text { experience }\end{array}$ \\
\hline Industry liaison opportunities & Too focused \\
\hline $\begin{array}{l}\text { Specific targeted content very } \\
\text { relevant to employers }\end{array}$ & $\begin{array}{c}\text { Industry partnership } \\
\text { inadequate or unstable }\end{array}$ \\
\hline $\begin{array}{l}\text { Adjunct to education programs offering } \\
\text { verifiable testing of skills and knowledge }\end{array}$ & $\begin{array}{l}\text { Training oriented rather than } \\
\text { education oriented }\end{array}$ \\
\hline $\begin{array}{c}\text { Potential employment advantages } \\
\text { for graduates }\end{array}$ & $\begin{array}{l}\text { "Value-for-Money" ignorance of } \\
\text { certification }\end{array}$ \\
\hline Precursor to licensing requirements & Too market and popularity driven \\
\hline
\end{tabular}

The advantage of training is that it provides usable knowledge linked to industry but it is also has disadvantages. For example, it focuses on one topic too narrow, hence students do not get a wider view of the problems and their knowledge soon becomes out of date. Another limitation of industrial training relates to the duration of the courses. The length of training cannot be changed according to student interest. It is also a pitfall that there is no course which combines the theory with the practice of ITIL. Preparation courses contain only theory while software companies focus only on teaching their own software. For instance,

"Universities should be preparing graduates for a career - not just a job. For students, there are advantages in learning about IT service management at university rather than industry training." [14]

\section{Developing the course}

The course was developed for undergraduate IT students.

Many courses at Óbuda University cover different parts of ITIL. The foundational theory of ITIL and more practical courses based on the process of ITIL 


$$
\text { "tmcs-erdelyi" — 2010/11/14 — 21:59 — page } 239 \text { — \#11 }
$$

Practice based course of Information Technology Service Management for BSc students 239

are offered to students. IT Service Management can be chosen as a specialization on undergraduate programmes.

The course under consideration in this paper needs to meet the following requirements:

- Optional course in BSc;

- Focusing on a practical approach;

- Giving comprehensive knowledge and practical skills as well to students;

- One lecture and two practice class per week (altogether 14x3x45 minutes).

\subsection{Build of course}

The course starts with theory. The introduction contains the review of service science. The need of IT services is shown with practical examples. Incident and problem management beginning in service desk and IT system administration are focused mainly in the review of ITIL's best practise. There is no possibility to demonstrate all topics related to IT service management neither in theory part nor in practical part of the syllabus. The processes and functions of ITIL are categorized into three groups according to the depth as being dealt with:

- In the first group there are functions whose targets and situations in the system are explained.

- Processes on the second group are examined more deeply by students. They have to solve so called brain storming tasks to get familiar with the certain process. Brain storming tasks may take longer or shorter time to solve and students can work alone or in groups during the class. The tasks are ended up with global discussions. An example for brain storming: "Find an IT Service. How can you be the best? Define your strategy."

- There are monitoring and configuration management in the third group. These topics are presented by means of software. Students use software in virtual environment specially formed to the topics. In both topics two software products are presented (one is proprietary and the other is open source) so that students can understand not only the software but the theory under it. Related protocols are also demonstrated to widen students' angle of view.

Table 2 shows depth of ITIL processes as they are taught. The shaded area indicates whether the process is discussed in the given level. 
Table 2. Depth of ITIL processes as they are taught

\begin{tabular}{|c|c|c|c|c|}
\hline Lifecycle & Process, Function or Activity & Explained & $\begin{array}{c}\text { Deeply } \\
\text { Discussed }\end{array}$ & $\begin{array}{c}\text { With } \\
\text { Software }\end{array}$ \\
\hline \multirow[t]{3}{*}{ Service Strategy } & Service Portfolio Management & & & \\
\hline & Demand Management & & & \\
\hline & IT Financial Managementt & & & \\
\hline \multirow[t]{10}{*}{ Service Design } & Service Catalogue Management & & & \\
\hline & Service Level Management & & & \\
\hline & Risk Management & & & \\
\hline & Capacity Management & & & \\
\hline & Availability Management & & & \\
\hline & IT Service Continuity Management & & & \\
\hline & Information Security Management & & & \\
\hline & Compliance Management & & & \\
\hline & IT Architecture Management & & & \\
\hline & Supplier Management & & & \\
\hline \multirow[t]{6}{*}{ Service Transition } & Service Asset and Configuration M. & & & \\
\hline & Service Validation and Testing & & & \\
\hline & Evaluation & & & \\
\hline & Release and Deployment M. & & & \\
\hline & Change Management & & & \\
\hline & Knowledge Management & & & \\
\hline \multirow[t]{7}{*}{ Service Operation } & Event Management & & & \\
\hline & Incident Management & & & \\
\hline & Problem Management & & & \\
\hline & Request Fulfillment & & & \\
\hline & Access Management & & & \\
\hline & Service Desk & & & \\
\hline & Monitoring & & & \\
\hline \multirow{2}{*}{$\begin{array}{l}\text { Continual Service } \\
\text { Improvement }\end{array}$} & Service Measurement and Reporting & & & \\
\hline & Continual Service Improvement & & & \\
\hline
\end{tabular}

Common tasks appear in Service Operation among ITIL Lifecycles: "Service Operation is where the strategy, design, transitions and improvements are delivered and supported on a day-to-day basis" [6]. It contains help desk function, incident, problem and change management process and monitoring activity. These tasks have to be handled in a workplace by a graduate employee. This is why many processes from Service Operation are chosen into the topics of the course.

Figure 5 shows the topics of IT service management course.

The purpose of choosing the topics was the importance of the topics and their usability. Monitoring and configuration management can also form the subjects of stand-alone courses. Monitoring of networks, workstations and servers is an 


$$
\text { "tmcs-erdelyi" — 2010/11/14 — 21:59 — page } 241 \text { — \#13 }
$$

Practice based course of Information Technology Service Management for BSc students

\begin{tabular}{|c|c|c|c|}
\hline \multirow{2}{*}{$\begin{array}{c}\text { Config. Mngment } \\
\text { Protocols }\end{array}$} & SW Mngment & Service Desk & \multicolumn{2}{|c|}{$\begin{array}{c}\text { Monitoring } \\
\text { Software }\end{array}$} \\
\hline Misc. & \multicolumn{2}{|c|}{$\begin{array}{c}\text { Service Transition Service Operation } \\
\text { ITIL }\end{array}$} & Misc. \\
\hline \multicolumn{3}{|c|}{ IT Service } \\
\hline
\end{tabular}

Figure 5. Syllabus of IT Service Management

established practice. Software management is solved with remote installation of software in almost every large enterprise. The method of assessment is shown on the webpage [15].

\subsection{Teaching of monitoring}

Monitoring networks, servers and workstations and the software on them is an everyday task for administrators for a long time. Logging events is a crucial function in complicated software. Special software developed for monitoring purposes has a capability more than only reading log files. It handles data of more devices in one place and values and events that can be given according to the interest of operators. Thresholds can be set in a good monitoring system. When the value crosses a threshold program alerts are automated and pay attention to new incidents. After alerting the program can execute commands to repair the problem. Reports from collected data by monitoring software help managers to effectively determine the mainstream of development of the company.

Standards are given to monitoring, retrieving and storing data. Distributed Management Task Force created the Common Information Model (CIM) and Web-Based Enterprise Management (WBEM). CIM determines the description of the handled element and the relationship between them. WBEM defines protocol for interoperation of system manager component by using CIM. Microsoft Windows Management Instrumentation (WMI) was developed by using CIM and WBEM standards. Information about components of operation systems can be gained by WMI. For Linux systems management technologies are developed within the confines of Standards Based Linux Instrumentation (SBLIM). Monitoring network devices are standardised by considering Simple Network Management Protocol (SNMP). 


$$
\text { "tmcs-erdelyi" — 2010/11/14 — 21:59 — page } 242 \text { — \#14 }
$$

There is a wide range of monitoring software. In some of them administrators need to know the standards mentioned above to take the appropriate settings. Other monitoring programs hide standards and provide own editors and inbuilt monitoring settings. Software in latter group usually is more complicated and provides other features (such as alerting, reports etc.). The best way is when students have opportunities to get to know more types of monitoring software. It is necessary for them to understand the basic and the root of the monitoring software (so they know how to set monitors according to standards and protocols). But students also need to be familiarised with more modern and versatile software. To choose the most appropriate programs for students the following aspects must be considered (the order of the items shows their importance):

- The subject of monitoring (network, server, workstation).

- System requirements.

- Support and documentation.

- Existence of trail version or free of charge software.

- The way of configuration (via files, with web interface).

- Work on multiplatform.

- Reporting and analysis.

- Event alert.

- Program management, remediation (execute commands).

- Discovering new systems.

Support and documentation are important because of self-study of students. Documentation in a good quality is necessary at least in English.

Students generally use virtual machines to form a small network and to practise monitoring tasks, hence system requirement of software is important.

During developing the syllabus it was assumed that students are familiar with log files and how to manage them, hence focusing upon the review of standards and the use of software. Beyond different types of configuration settings monitoring topics consist of setting events and thresholds, problem management and tasks related to reporting business data. Figure 6 shows the topics of monitoring.

If there is enough time and students are interested in programming then there is a possibility to teach how to write programs that can be monitored, namely to write programs that provide information about their own operations. 


$$
\text { "tmcs-erdelyi" — 2010/11/14 — 21:59 — page } 243 \text { — \#15 }
$$

\begin{tabular}{|c|c|}
\cline { 2 - 2 } \multicolumn{1}{c|}{} & Events \\
Thresholds \\
Alerts \\
Problem Management \\
\hline Creating & Setting with user interface \\
Setting & Setting by standards \\
Converting & SNMP, CIM, WBEM, WMI \\
Reports & Monitoring Settings \\
\hline
\end{tabular}

Figure 6. Topics of monitoring

\subsection{Teaching of configuration management}

Creating a configuration management database covering all data are not realized currently in enterprises. However automated remote deployment software products may show a good example how to collect automatically and use information. These applications collect hardware and software information about discovered computers and manage licenses and patches. It is possible to determine which software to be installed in which computer according to hardware and software information.

While the need for automated remote deployment pre-existed many technologies are used to implement it. The simplest devices are script-based tools that start an installation and simulate the appropriate mouse moves and key strokes on the target machine. The next step of the evolution of the technology when the client machine turns to the server at every launch to get the list of software that should be on the client. If one piece of software is not installed on a client, the deployment starts. (In this case the installation software package should be configured for unattended deployment.) In state-of-the-art solutions an agent is installed to the target machine which follows the instruction of the server. In this case software deployment can happened any time and a database on the server stores all the information about software on the target machine. The latest technology is the closest to the conception of configuration management. These types of applications provide the possibilities to licence management and patch management.

Specific software packages are needed for unattended software development. Creating such packages is an interesting topic but only its simplest version fits to 


$$
\text { "tmcs-erdelyi" — 2010/11/14 — 21:59 — page } 244 \text { — \#16 }
$$

the syllabus. In this case students use the inbuilt possibilities. There are methods to repackage software totally but it goes beyond the limitations of the syllabus.

To choose the most appropriate programs for students the following aspects must be considered (the order of the items shows their importance):

- The way of configuration (via files, with web interface).

- System requirements.

- Support and documentation.

- Existence of trail version or free of charge software.

- Work on multiplatform.

- Existence of inventory features.

- Managing licenses and patches.

The most emphatic technology in the teaching of configuration management is the agent-server based technology because it uses the core concepts of configuration management. But if there is enough time, it is beneficial to demonstrate the other technologies. Figure 7 shows the topics of configuration management course [16]. The syllabus is shown on the webpage [15].

\begin{tabular}{|c|c|c|}
\multicolumn{1}{c|}{} & Technologies of OS Depl. & \multicolumn{1}{|c}{} \\
\cline { 2 - 3 } & Configuration Management & \\
\hline Remote & Invetntory Mngment & Creating \\
OS & Software Mngment & Software \\
Deployment & Licence Mngment & Packages \\
\hline
\end{tabular}

Figure \%. Topics of configuration management

\section{Conclusion}

The course was taught in both semesters for the academic year 2009/2010. Feedback from students was positive coming from formal and informal consultation. In theory part 17 processes of ITIL were presented to the students and 8 out of them were demonstrated elaborately. The theory part was made more diversified by so called brain storming tasks which were solved with enthusiasm 
and sometimes very thoroughly by the students. Consequently, students joined their tasks related to development and implementation of IT services with their projects in other courses. The practical topic monitoring section took more time than expected (presentation and use of two software products and application of protocols) so there was less time for the topic of configuration management. But it did not show serious limitations. To summarise, the course managed to provide students with the correct knowledge and assessment.

\section{References}

[1] J. van Bon, editor, IT Service Management: An Introduction, Van Haren Publishing, 2002.

[2] Wikipedia, Control objectives for information and related technology (last visit 22.04.10), http://en.wikipedia.org/wiki/COBIT.

[3] Wikipedia, Information technology infrastructure library (last visit 22.04.10), http://en.wikipedia.org/wiki/Information_Technology_Infrastructure_Library.

[4] Microsoft Technet, Microsoft Operations Framework (last visit 22.04.10), technet.microsoft.com/en-us/library/cc506049. aspx.

[5] The ITIL Wiki, Comparison between ITIL V3 and ITIL V2 (last visit 23.04.10), http://wiki.en.it-processmaps.com/index.php/Comparison_between_ITIL_V3_and_ ITIL_V2_-_The_Main_Changes.

[6] Office of Government Commerce, Service Operation Book (ITIL), ISBN-13: 978-0113310463.

[7] Office of Government Commerce, Service Strategy Book (ITIL), ISBN-13: 978-0113310456.

[8] T. Bishop, CTO BMC Software: Why You Need a Configuration Management Database: Where ITIL Fits (last visit 12.05.09), http://talk.bmc.com/docs/v2bmc-itil-cmdb-bishop1.ppt.

[9] B. C. Potgieter, J. H. Botha and C. Lew, Evidence that use of the ITIL framework is effective, 18th Annual Conference of the National Advisory Committee on Computing Qualifications, 2005, 124-131.

[10] A. Cater-Steel and M. Toleman, The Role of Universities in IT Service Management Education, 11th Pacific-Asia Conference on Information Systems, 2007, 369-382.

[11] S. Galup, R. Dattero, J. J. Quan and S. Conger, Information Technology Service Management: An Emerging Area for Academic Research and Pedagogical Development, SIGMIS-CPR'07, 2007, 46-52.

[12] G. Fazekas, Syllabus of Információs rendszerek menedzselése on University of Debrecen, http://www.inf.unideb.hu/ fazekasg/oktatas/INFORMRENDSZEREK_MENEDZS.pdf. 


$$
\text { "tmcs-erdelyi" — 2010/11/14 — 21:59 — page } 246 \text { — \#18 }
$$

[13] Jovanovic, Bentley, Stein and Nikakis, Implementing industry certification in an is curriculum: An Australian Experience, Information Systems Education Journal 4(59) (2006), ISSN=1545-679X.

[14] A. Cater-Steel and M. Toleman, Education for IT service management standards, International Journal of IT Standards and Standardization Research 5(2) (2007), 27-41, ISSN=1539-3062.

[15] K. Erdélyi, Syllabus of the course, http://nik.uni-obuda.hu/oktig/kovetelmenyek10tavasz/NSTBS1SERB_VL .doc.

[16] L. Zsakó, Informatikai kompetenciák, avagy miért tanítunk informatikát?, INFO ÉRA 2008 Az informatikaoktatás szabályozása és módszertana a közoktatásban Published on CD, 2008.

KRISZTINA ERDÉLYI

ÓBUDA UNIVERSITY

HUNGARY

E-mail: erdelyi.krisztina@nik.uni-obuda.hu

(Received November, 2009) 